\title{
Gender differences in animal models of posttraumatic stress disorder
}

\author{
Hagit Cohen ${ }^{\mathrm{a}, *}$ and Rachel Yehuda ${ }^{\mathrm{b}}$ \\ ${ }^{a}$ Ministry of Health Mental Health Center, Anxiety and Stress Research Unit, Faculty of Health Sciences, \\ Ben-Gurion University of the Negev, Beer-Sheva, Israel \\ ${ }^{\mathrm{b}}$ Post-Traumatic Stress Disorder Program, Bronx VA Medical Center, Mount Sinai School of Medicine, Bronx, NY, \\ USA
}

\begin{abstract}
Epidemiological studies report higher prevalence rates of stress-related disorders such as acute stress disorder and post-traumatic stress disorder (PTSD) in women than in men following exposure to trauma. It is still not clear whether this greater prevalence in woman reflects a greater vulnerability to stress-related psychopathology. A number of individual and trauma-related characteristics have been hypothesized to contribute to these gender differences in physiological and psychological responses to trauma, differences in appraisal, interpretation or experience of threat, coping style or social support. In this context, the use of an animal model for PTSD to analyze some of these gender-related differences may be of particular utility. Animal models of PTSD offer the opportunity to distinguish between biological and socio-cultural factors, which so often enter the discussion about gender differences in PTSD prevalence.

In this review, we present and discuss sex-differences in behavioral, neurochemical, neurobiological and pharmacological findings that we have collected from several different animal studies related to both basal conditions and stress responses. These models have used different paradigms and have elicited a range of behavioral and physiological manifestations associated with gender. The overall data presented demonstrate that male animals are significantly more vulnerable to acute and chronic stress, whereas females are far more resilient. The stark contradiction between these findings and contemporary epidemiological data regarding human subjects is worthy of further study. The examination of these gender-related differences can deepen our understanding of the risk or the pathophysiology of stress-related disorders.
\end{abstract}

Keywords: Post traumatic stress disorder, animal model, HPA-Axis, corticosterone, sympathoadrenal system, estrogen, resilience, vulnerability

\section{Introduction}

Virtually every large scale epidemiologic study has documented a greater prevalence of posttraumatic stress disorder (PTSD) in women than men. However, it is still not clear whether this greater prevalence in woman reflects a greater vulnerability to stress-related psychopathology [1]. The alternative explanation is that the greater prevalence of PTSD reflects the reality in our society that woman are likely to be more vic-

*Address for correspondence: Hagit Cohen, Ph.D., Anxiety and Stress Research Unit, Ministry of Health Mental Health Center, Faculty of Health Sciences, Ben-Gurion University of the Negev, P.O. Box 4600, Beer-Sheva 84170 Israel. Tel.: +972 8 6401743; Fax: +972 8 6401742; E-mail: hagitc@bgu.ac.il. timized by the types of events that result in PTSD than men, and that they further may experience these events differently [2]. Indeed it has been somewhat difficult to study the nature of the gender difference in PTSD because what might seem a similar exposure in men and women (i.e., assault), may be interpreted and experienced differently by men and women. Nonetheless, Breslau et al. [3] reported that the overall conditional vulnerability for PTSD among those exposed to a potential traumatic experience (PTE) is approximately twofold higher in women than men, adjusting for gender difference in the distribution of type of trauma. This group demonstrated that with respect to overall lifetime trauma, men experienced more events than women. However, Robin et al. [4] reported higher lifetime prevalence of trauma exposure in women than in men. 
Other studies [5] have found that the predictive validity of gender is limited in the context of trauma exposure. In a prospective study of PTSD, Freedman et al. [6] reported that no gender-related differences were found in response to motor vehicle accidents. Men and women showed similar recovery rates from PTSD and associated symptoms. These data suggest that retrospective reports of trauma exposure or PTSD may be subject to gender differences in recall bias or attribution of lifetime events as traumatic [6]. Yasan et al. [7] also found no gender-related differences in PTSD prevalence among people living within an area of conflict, but reported that there was a different risk of PTSD among men and women who experienced similar traumatic events: the risk of PTSD for those who experienced military conflict was higher among men then woman. Similarly, the gender differences in PTSD in men is reportedly higher $(65 \%)$ than in women $(45 \%)$, though this trauma occurs more than 10 times more frequently in women [8]. In contrast, however, the magnitude of the gender difference in PTSD prevalence yielded an odds ratio of approximately 5 when assault was included. However, this ratio dropped to 3 when sexual trauma was excluded from calculations [9]. It is reasonable to conclude from the above discussion that some gender differences in PTSD prevalence may in part be related to gender differences in trauma exposure, but some may reflect differences in genderspecific PTSD risk factors. It seems important to also consider that different conclusions might be reached from retrospective vs. prospective studies. In this context, the use of an animal model for PTSD to analyze gender differences may be of particular utility. Animal models of PTSD further offer the opportunity to distinguish between social and cultural factors, which so often enter the discussion about gender differences in PTSD prevalence, and biological alterations which might contribute to gender.

In considering gender differences in PTSD biology that could help guide our understanding of PTSD prevalence, the data are limited. Some gender differences have been noted, however. For example, studies of cortisol have shown that levels of salivary cortisol increase in males over the course of a day, while levels decrease among females [10], and that among children with PTSD symptoms, females have significantly elevated cortisol levels compared to males [11]. Differences in catecholamines have also been observed: among males epinephrine and norepinephrine are associated with PTSD symptoms 1 month and 5 months following trauma, but the same is not true for females [12].
A number of studies have identified some neuroanatomical differences in males and females with PTSD. Magnetic Resonance Imaging (MRI) of the brains of children with PTSD due to maltreatment show decreased intracranial and cerebral volumes among than controls, with greater corpus callosum reduction (and a trend for greater cerebral volume reduction) in males than females [13]; larger prefrontal lobe cerebro-spinal fluid volumes and smaller splenium compared to controls which is more prominent in males; and smaller cerebral volumes and corpus callosum regions 1 (rostrum) and 6 (isthmus) and greater lateral ventricular volume increases in males than females with PTSD [14]. Functional MRI (fMRI) has also illustrated that among males, enhanced brainstem activity is associated specifically with the diagnosis of PTSD, but that among females such enhanced activity is associated with exposure to trauma (but not necessarily development of PTSD [15]. In this same study, it was observed that males with PTSD displayed greater hippocampal activity to fear than females with PTSD. However, what is true for all of these studies is that the gender differences observed in men and women with PTSD may simply reflect normal gender differences in these measures that are do not speak to, or reflect, differences in PTSD risk or psychopathology in men and women. That is, that there may be normal gender differences because men and women differ on many parameters. A gender differences that can help explain greater PTSD prevalence or response to trauma is arguably one in which there is a gender difference in PTSD which is not otherwise observed (i.e., a gender $\mathrm{x}$ diagnosis interaction).

It is for this reason, that an analysis of gender differences in animal models of PTSD may be extremely informative. Animal models of PTSD, despite their limitations, provide a fair approximation of certain aspects of this complex clinical disorder, enabling the study of questions raised in clinical research, with the advantage of prospective study designs, under far more controllable conditions. Animal models of PTSD can thus represent a useful tool for the investigation of sex differences in the neurobiology of PTSD.

Unfortunately, the amount of information regarding the gender-related differences in animal models of PTSD is also very limited to date. The majority of animal-based studies of PTSD have used male animals. A limited number have used females. Presumably, this tendency has stemmed mainly from the desire to preclude any confounding effects that female genderrelated factors may have on the results, e.g. the estrous cycle. Virtually no studies have been performed 
which intentionally and systematically attempt to compare male vs. female animals.

In this review, we present and discuss sex differences in behavioral, neurochemical, neurobiological and pharmacological findings that we have collected from several different animal studies related to both basal conditions and stress responses. These models have used different paradigms and elicited a range of behavioral and physiological manifestations associated with gender. Overall the results have been interesting and indicate that further gender-related animal studies may well provide useful information.

\section{Gender differences under resting conditions (unstressed)}

\subsection{Gender differences in basal HPA-axis activity}

In mature rats, sex-related differences in pituitary and adrenal function have been reported quite extensively, with generally higher plasma corticosterone concentrations observed in females during rest [16-22]. Critchlow et al. [21] reported sex-related differences in the circadian rhythm of adrenal cortical function between males and females. Although both sexes showed evidence of a 24-hr rhythm in plasma and adrenal corticosteroids, the maximal levels observed and the mean 24-hr concentrations were markedly higher in females with mature ovaries than in males.

The gender differences in HPA-axis function are in part due to differences in the circulating gonadal steroid hormone milieu. The higher levels of plasma and adrenal corticosterone that were observed at proestrus suggest that estrogens may be primarily responsible for the female type circadian pattern. In both sexes, estrogen administration increases basal corticosterone levels $[23,24]$. To further explore the possibility of sex differences in pituitary-adrenal function under resting conditions, Critchlow et al. [21] demonstrated that findings in castrated and prepubertal animals implicate the ovary in mechanisms responsible for the sex differences in the circadian rhythm of adrenal cortical function. Both castrated and prepubertal females failed to show the marked excursions of plasma and adrenal corticosterone that were present in intact and sham-castrated females. Moreover, the lower resting levels of plasma and adrenal corticosterone that were observed following ovariectomy were manifested in the absence of a change in adrenal weight. Spayed females, like intact and sham-operated females, had adrenals that were found to be heavier than those found in males. Taken together, the cyclic variations in ovarian activity apparently lead to marked differences in corticosteroid secretion.

\subsection{Gender differences in structures and functions in the CNS}

Hormones organize the neuronal circuitry involved in neuroendocrine functions and behavior, and are responsible for gender differences in the size of certain brain areas, synaptic connections, neurotransmitter concentrations and their activity [25,26]. For example, in the late 1970s, a nucleus in the anterior hypothalamus was discovered to be 2.5-5 times larger in males than females [27]. This nucleus is known as the sexually dimorphic nucleus of the medial preoptic area, and plays an important role in mediating the display of male sexual behavior [28]. The larger size in males has been attributed to prevention of neuronal apoptosis by circulating testosterone after its conversion to estradiol [29]. In males but not in females, Krieg's areas 2 and 3 on the right side of the cerebral cortex are thicker than on the left side [30,31]. On the other hand, the rostral anterior commissure, a fiber tract connecting the left and right hemispheres and several brain regions including the lateral amygdala nucleus, accumbens and endopiriform cortex, is larger in females than in males [32].

At the morphological level, the hippocampal formation also has several sexual dimorphisms. In the pyramidal cell layers of the hippocampus, the field volumes and cell bodies of CA1 and CA3 pyramidal cells are larger in males than females [33,34]. Male rats also have more glial fibrillary acidic protein immunoreactive (GFAP-IR) astrocytes in the CA3 region [35], greater dendritic branching in the $\mathrm{CA} 1$ region of the stratum radiatum (the dendritic field of the apical dendrites of the CA1 pyramidal cells) [36] and more apical dendritic excrescences in the stratum lucidum than female rats [37]. Additionally, males have a larger dentate gyrus (DG) [38,39] and greater synaptic connectivity than females [40]. Furthermore, the subiculum, the source of major hippocampal efferents, is larger in males compared with females [41]. In contrast, females have more GFAP-IR astrocytes in the CA1 region [35] and a greater number of primary dendrites in the CA3 region than males [37].

The naturally occurring fluctuation in estrogens that occurs across the estrous cycle can dramatically influence the morphology of the hippocampus in female rats. Females in the proestrous cycle stage (i.e. when estro- 
gen levels are at their peak) have higher levels of dendritic spines in the CA1 region compared to males [42]. Between proestrus and estrus, when estrogen levels fall, there is a $30 \%$ decrease in spine density; a comparable decrease in synapse density at the electron microscope level is the ultra-structural reflection of the spine density change [43]. Reducing estradiol levels by ovariectomy causes a decrease in spine density (Gould et al., 1990), and estradiol replacement increases spine density, spine size and excitatory synapse number, and enhances LTP amplitude in hippocampal CA1 pyramidal neurons in ovariectomized rats [43-47]. No comparable change was found in CA3 or the DG. Estrogen also increases the occurrence of multi-synaptic boutons, which are presynaptic elements that make more than a single synapse at the apparent expense of singlesynaptic boutons [48]. This suggests that during the portion of the estrous cycle when estrogen levels are at their highest, new dendritic spines make synapses with preexisting boutons. More recently, it has been found that the synapses of a multisynaptic bouton are made with different postsynaptic neurons [49], which indicates not only an increase in spine and synapse number, but also in divergence of inputs [50]. Further studies have demonstrated that a variety of synaptic markers are also upregulated after systemic estrogen application [51], confirming the supportive role of estrogens for synapse formation. The results of these studies on estrogen-induced synaptic plasticity and the fact that gonads are the main source of estrogen strongly suggest an endocrine regulation of spinogenesis in the hippocampus by gonadal estrogen. Interestingly, estrogen increases CA1 spine density in females but not in males [52,53]. Castration reduces CA1 spine density in males, but estrogen treatment does not reverse this decrease [52]. The molecular mechanisms through which estrogen fails to induce spinogenesis in males are not fully understood.

The molecular mechanisms controlling estrogeninduced pyramidal neuronal dendrite and spine morphogenesis are being elucidated. Estrogen increases the expression of NMDA receptors in the stratum oriens and stratum radiatum of the CA1 pyramidal cells, which is necessary for estrogen-induced spine formation [54,55]. Females who are treated simultaneously with NMDA receptor antagonists and estradiol fail to show estrogen-induced increases in hippocampal spine density [56].

These gender differences in morphology further suggest a mechanism for how similar exposures might result in differential outcomes in men and women. These mechanisms and potential brain differences should be more fully examined in both traumatized and nontraumatized men and women.

\subsection{Gender differences in the sympathoadrenal system}

Weinstock et al. [18] reported that female rats show greater individual variation than males in their concentrations of plasma catechols and corticosterone, and have higher basal levels of plasma corticosterone, norepinephrine (NE) and dihydroxyphenylacetic acid (DOPAC). On the other hand, female rats have a lower plasma dihydroxyphenylglycol(DHPG)/NE ratio (DHPG is derived from released NE that has undergone reuptake into the neuron, and also from some leakage from storage vesicles), which suggests that proportionally less NE is taken back into nerve terminals than in males. Furthermore, adult males have significantly higher mean arterial blood pressure (MAP) than females [18].

In sum, under basal conditions, sex-related differences in pituitary and adrenal function have been reported quite extensively. Moreover, morphological gender differences in brain areas may explain sex differences in function. It is well known that the brainpituitary-adrenal axis plays an important role in mediating an animal's ability to cope with stress [57]. It is therefore possible that the basal sex-related differences may influence the ability of the organism to respond to, cope with and adapt to stressful stimuli, and therefore may have an influence on stress-induced disease susceptibility

\section{Gender differences in response to stressors}

Gender-related differences in the stress response in the animal literature have been noted for more than 40 years. So the question becomes, in translating this work to PTSD, whether any gender differences in PTSD are simply a reflection of normal differences between the genders, and if so, what the pathophysiologic and treatment implications of these gender differences might be.

\subsection{Gender differences in HPA-axis reactivity}

Gender differences in the response of the HPA-axis to a variety of stressors have been demonstrated in multiple animal species [24]. Endocrinology studies are 
beginning to reveal fairly consistent data indicating that female rats demonstrate markedly higher and more persistently increased plasma levels of corticosterone after stress exposure (physical and psychological) than do male animals [18,22,58-60]. Louvart et al. [59] reported that female rats react to a physical stressor with an endocrine response that is quantitatively greater and longer than the response of the males. Forty-two days after a traumatic procedure (an intense inescapable footshock followed by situational reminders), female rats exhibited increased negative feedback of the HPAaxis, whereas no effect was seen in male animals [59]. Additionally, circulating corticosteroid-binding globulin (CBG) levels after stress are approximately twofold higher in adult female rats compared with males [61]. Female rats also show greater adrenocorticotrophin (ACTH) responses to stress [62] than males. Several hypotheses have been suggested to explain these data: 1) There is a gender-related difference in the responsiveness of the adrenal gland to an equivalent amount of ACTH stimulation. 2) There is a difference in the rate of clearance and metabolism of plasma corticosterone between male and female rats. 3) There is a sex difference in the amount of ACTH released in response to stress. 4) These gender differences suggest that postpubertal gonadal steroid hormone secretion may play an important role in the activation of the stress response.

Plasma corticosterone concentrations following intraperitoneal injection of ACTH or saline were measured to explore the first of these hypotheses [22]. Female rats demonstrated markedly higher and more persistently increased plasma levels of corticosterone after ACTH injection than did male rats. The data support the hypothesis that the adrenal gland of the female rat is more sensitive to ACTH than is the male adrenal gland [22]. Kitay et al. [22] proposed an alternative explanation, equally applicable to the effects obtained after stress or after ACTH administration: that a slower rate of plasma clearance and metabolism of corticosterone occurs in female rats.

To explore the second hypothesis, the clearance (biological half-life) of injected corticosterone was evaluated in male and female rats; they were injected 16 hours after adrenalectomy to eliminate variation due to endogenous steroid secretion [22]. The biological halflife in male rats was 20 minutes, whereas the comparable value in female animals was 13 minutes [22,63]. Interestingly, no sex difference was observed in the rate of reduction of the sidechain of corticosterone [22]. Finally, the concentration of corticosterone in the adrenal vein of the female rat is 2.5 times that in the male. This finding may not be correlated with a sex difference in adrenal size since adrenal weights were approximately equal. Thus, the higher plasma corticosterone levels observed after ACTH or stress in female rats are not explained by sex differences in the rate of clearance and metabolism of the steroid [22].

In contrast to these findings, sex differences in the metabolism of cortisone by rat liver homogenates have been reported by Hagen and Troop $[64,65]$. Comparison of hepatic metabolism of steroid between genders showed that liver tissue from female rats reduces the $\mathrm{A}$ ring of both cortisone and corticosterone more rapidly than does male liver tissue [63-66]. Conversely, the C20 ketone of cortisone is metabolized more effectively by male liver tissue $[64,65]$.

The third possibility to be considered is that the amount of ACTH released in response to stress differs between the genders. In other words, the increased responsiveness of the female adrenal cortex may be associated with increased responsiveness of the female pituitary gland with respect to ACTH secretion [22]. In footshock stress, females show higher and more rapid secretion of ACTH than males [16,67]. Moreover, Gemzell has reported increased secretion of ACTH in male rats following administration of estradiol [68]. The authors suggested that estradiol affects both the hypophysial secretion of ACTH and adrenal cortical sensitivity to ACTH based upon adrenal weight changes in intact and castrated rats [69].

\subsection{Role of estrogen}

The effect of estrogen on ACTH secretion is manifested as a prolongation of secretion [23]. This suggests that one mechanism by which estrogen enhances stress hormone secretion is by impairing glucocorticoid receptor (GR)-mediated negative feedback, as the increased responsiveness to a stressor that is seen in females is likely due to decreased sensitivity to glucocorticoid feedback [23,70-74]. Recently, Weiser and Handa [75] reported that estradiol exerts its action at the level of the paraventricular nucleus of the hypothalamus $(\mathrm{PVN})$, via estrogen receptor alpha $(\mathrm{ER} \alpha)$-mediated disruption of GR-mediated negative feedback.

In contrast, in studies with gonadectomized or neonatally estrogenized rats, Patchev et al. [76] and McCormick et al. [77] reported significantly higher HPAaxis response to stress in females independent of differences in circulating gonadal steroid levels, which suggests an innate or organized difference in the HPAaxis response to stress. Such organizational effects de- 
scribe the ability of gonadal steroids to program, early in development, subsequent central nervous system responses to gonadal steroids later in life $[78,79]$.

Taken together, these studies suggest that in addition to changes in neuronal function, estrogen can modulate the HPA-axis at multiple levels, from genomic differences to organizationally or developmentally programmed effects (caused by earlier differential gonadal steroid exposure), and/or acute, activational effects of recent gonadal steroid exposure.

\subsection{Role of androgen}

Gaskin and Kitay [80] demonstrated that the gonads influence hypothalamic and pituitary regulation of adrenocortical function in the hamster. In the male, exogenous testosterone enhances ACTH secretion, leading secondarily to increased adrenal steroid secretion and increased hepatic metabolism of cortisol. These data suggest that testosterone normally acts to inhibit the HPA response to environment perturbation.

Handa et al. [81] studied the role of androgens in the regulation of ACTH and corticosterone responses to foot shock and novelty stressors in gonadectomized or intact male F344 rats. They reported that castration enhances and androgen treatment suppresses the ACTH and corticosterone responses to a physical or psychological stressor. This effect of androgen is mediated via an androgen receptor mechanism and does not appear to involve changes in anterior pituitary sensitivity to $\mathrm{CRH}$ or to changes in hippocampal or hypothalamic corticosterone receptor concentrations [81]. Moreover, the effect of castration can be inhibited by administration of the non-aromatizable androgen, dihydrotestosterone, thus implicating androgen receptors in this regulatory pathway. In addition, Bingaman et al. [82] have demonstrated that long term (10 days) following castration, there are increases in hypothalamic CRH content and CRH-IR cell numbers in the PVN due to removal of androgen-dependent repression. These data suggest that the effect of androgen on HPA activity can be mediated via changes in CRH-containing cells within the PVN [24].

In sum, the results from a variety of studies suggest that gender differences in the ACTH and corticosterone response to stressors are a consequence of differences in gonadal steroid hormone secretion and their effects on neuronal functioning. An evolutionary explanation suggests that this sexually dimorphic regulation is an attempt to maintain reproductive competence in the face of physical or psychological insults that threaten homeostasis.

\subsection{Gender differences in sympathoadrenal system reactivity}

Weinstock et al. [18] reported that in response to stress exposure, there were significant sex-related differences in the extent and duration of the response of EPI and DHPG. A greater rise in plasma concentration of EPI was found in females than in males after exposure to footshock stress. This may indicate that the female adrenal gland is more reactive to the stress of a novel environment and footshock. Moreover, exposure to the shock box resulted in a significant increment in plasma DHPG in males and of DOPA and DOPAC in females. The finding that stress produces larger increments of plasma DOPA and DOPAC in female rats indicates that tyrosine hydroxylase in the sympathetic nerve terminals and adrenal medulla may also be higher than in males. A smaller DHPG/NE ratio was also found in females after stress, suggesting that neuronal uptake of NE is lower in females than in males [18]. Taken together, these data show that the female sympathoadrenal system is more reactive than that of the male to the stresses of a novel environment and footshock.

\subsection{Gender differences in molecular signaling}

Lin et al. [83] reported sex-related differences in the expression of $\mathrm{p}$-CREB in the prefrontal cortex (PFC) and DG after acute footshock stress. Acute stress caused a significant reduction in p-CREB immunoreactivity in a time-dependent manner in male DG two hours after exposure to the stress box. In contrast, acute stress had no significant effect on the expression of p-CREB in female PFC and DG.

Shansky et al. [84] asked whether ovariectomized females with estrogen replacement or vehicle treatment responded differently to stimulation of alpha- 2 adrenoceptors after administration of the benzodiazepine inverse agonist FG7142, a pharmacological stressor. The alpha-2 agonist, guanfacine, protected working memory from the impairing effects of FG7142 in the estrogen replacement group, but not in rats without replacement. No changes in alpha-2 receptor expression in the PFC were found, indicating that the behavioral effects observed were likely not due to changes in receptor expression. Thus, these findings point to possible mechanisms by which estrogen may enhance the stress response, and hold implications for the gender discrepancy in the prevalence of stress-related mental illness. 


\subsection{Gender differences in response to pharmacological treatments}

Poltyrev and Weinstock [85] have examined whether oral administration of amitriptyline for 6 weeks before puberty to prenatally stressed (PS) male and female rats could prevent hyper-anxiety in adulthood. They reported that early treatment with amitriptyline can prevent hyper-anxiety in PS rats in adulthood. Moreover, the anxiolytic effect was more readily seen in females than in males. The action of amitriptyline may differ in the two sexes because it is metabolized more rapidly in male than in female rats to nortriptyline by CYP2D in the liver [86].

\subsection{Prevalence rates of individual patterns of response in an animal model of PTSD}

Previously, we examined gender-related differences in the prevalence rates of individuals displaying a PTSD-like behavioral pattern in response to predator scent stress (PSS) exposure in male vs. female subjects [58]. An animal model that compares the behavioral response patterns of global populations to those of individual subjects was applied in a prospective comparative study of male rats and fertile female rats across the estrous cycle. The model classifies individuals within each study group according to the degree of their behavioral responses to a stressor in two sequential test paradigms (the elevated plus maze and the acoustic startle response), according to well defined and validated cutoff behavioral criteria (CBC's) [87-90]. Three classes of response pattern are defined - extreme behavioral response, minimal behavioral response and partial behavioral response - and prevalence rates of individuals displaying each pattern may then be calculated. Extreme responders display grossly disordered behaviors and are considered to most validly reflect/model PTSD-like responses [87-90].

Analysis of individual patterns of behavioral response to PSS exposure revealed that, although the global data for the entire population appeared to indicate that males and females responded differently, in fact the prevalence rate of severely affected animals was not significantly affected by gender (the prevalence of severely behaviorally affected female rats was $13.6 \%$, whereas in males it was 10\%). Although baseline levels of anxiety were higher for females and their peak levels of response to stress exposure were lower, the overall incidence of PTSD-like behavioral responses was the same for both genders.

\section{Conclusions}

The data presented above show that gender-related differences can be demonstrated on the phenotypic (behavior) and/or the endophenotypic (neurobiology) under baseline conditions and in response to stress. In adult rodents, gender-related differences in the regulation of the HPA-axis have been well established, with generally higher plasma corticosterone concentrations observed in females during rest and stress response. Significant differences have been reported regarding anatomical, morphological, and biochemical characteristics under baseline conditions and after stress exposure. This may reflect effects of gonadal steroids and could be the result of influences of sex hormones on neuroendocrine regulatory mechanisms and/or the modulatory action of circulating and CNS gonadal steroids. However, gender differences of the CNS and behavior may have an underlying genetic component, independent of any sex steroid-dependent mechanism. In addition, the environment can have a significant impact on the dimorphism and sexual differentiation of the CNS.

The data presented above demonstrate that male animals are significantly more vulnerable to acute and chronic stress, whereas females are far more resilient. But this, of course, is in stark contradiction to epidemiological data regarding the prevalence of PTSD in humans. Possibly, this gender-related difference between the patterns of vulnerability/resilience to posttraumatic pathology for humans and rodents (as well as in non-human primates) may stem from the difference in basal cortisol/corticosterone levels between males vs. females. In rodents the basal corticosterone levels of females are significantly higher than males and their response to stress is more adaptive [16-22]. In humans, the reverse is true: males display a higher basal cortisol level (during fertility years) associated with lower prevalence of stress-related psychopathology [91-95]. This might explain the disparity in findings in rodent and human studies. Translating the above work to PTSD, one might predict that higher basal cortisol levels might be protective for the development of PTSD. Indeed, it has been observed that lower cortisol levels were observed before and in the immediate aftermath of PTSD in those who subsequently developed PTSD [96]. It has been suggested that lower baseline cortisol is a pretraumatic risk factor for subsequent stress-related psychopathology [97].

This apparent paradox offers the possibility of examining gender differences in a manner where the iden- 
tification may be of significance in understanding risk or pathophysiology. Indeed, PTSD is not a normative response to stress because of its infrequent occurrence compared to the prevalence of trauma. If the normal biological and behavioral profile suggests that females should be more resilient, it is imperative to identify the biological paramaters in men and women that deviate from this normal pattern as these may ultimately be the correct targets of PTSD prophylaxis and intervention.

\section{References}

[1] M. Olff, W. Langeland, N. Draijer and B. Gersons, Gender differences in posttraumatic stress disorder, Psychol Bull 133 (2007), 183-204.

[2] L. Pratchett, M. Pelcovitz and R. Yehuda, Trauma and Violence: Are Women The Weaker Sex? Psychiatric Clinics of North America 33 (2010), 465-474.

[3] N. Breslau, H.D. Chilcoat, R.C. Kessler, E.L. Peterson and V.C. Lucia, Vulnerability to assaultive violence: further specification of the sex difference in post-traumatic stress disorder, Psychol Med 29 (1999), 813-821.

[4] R.W. Robin, B. Chester, J.K. Rasmussen, J.M. Jaranson and D. Goldman, Prevalence and characteristics of trauma and posttraumatic stress disorder in a southwestern American Indian community, Am J Psychiatry 154 (1997), 1582-1588.

[5] C.R. Brewin, B. Andrews and J.D. Valentine, Meta-analysis of risk factors for posttraumatic stress disorder in trauma-exposed adults, J Consult Clin Psychol 68 (2000), 748-766.

[6] S.A. Freedman, N. Gluck, R. Tuval-Mashiach, D. Brandes, T. Peri and A.Y. Shalev, Gender differences in responses to traumatic events: a prospective study, J Trauma Stress 15 (2002), 407-413.

[7] A. Yasan, G. Saka, M. Ozkan and M. Ertem, Trauma type, gender, and risk of PTSD in a region within an area of conflict, J Trauma Stress 22 (2009), 663-666.

[8] R.C. Kessler, A. Sonnega, E. Bromet, M. Hughes and C.B. Nelson, Posttraumatic stress disorder in the National Comorbidity Survey, Arch Gen Psychiatry 52 (1995), 1048-1060.

[9] M. Stein, J. Walker and D. Forde, Gender differences in susceptibility to posttraumatic stress disorder, Behav Res Ther $\mathbf{3 8}$ (2000), 619-628.

[10] B. Freidenberg, R. Gusmano, E. Hickling, E. Blanchard, J. Bremner and C. Frye, Women with PTSD have lower basal salivary levels later in the day than do men with PTSD: a preliminary study, Physiol Behav 99 (2010), 234-236.

[11] V. Carrion, C. Weems, R. Ray, B. Glaser, D. Hessl and A. Reiss, Diurnal salivary cortisol in pediatric posttraumatic stress disorder, Biol Psychiatry 51 (2002), 575-582.

[12] L. Hawk, A. Dougall, R. Ursano and A. Baum, Urinary catecholamines and recent-onset posttraumatic stress disorder after motor vehicle accidents, Psychosom Med 62 (2000), 423434.

[13] M. DeBellis, M. Keshavan, D. Clark, B. Casey, J. Giedd, A. Boring, K. Frustaci and N. Ryan, A.E. Bennett Research Award. Developmental traumatology. Part II: Brain development, Biol Psychiatry 45 (1999), 1271-1284.

[14] M. De Bellis and M. Kehavan, Sex differences in brain maturation in maltreatment-related pediatric posttraumatic stress disorder, Neurosci Biobehav Rev 27 (2003), 103-117.
[15] K. Felmingham, L. Williams, A. Kemp, B. Liddell, E. Falconer, A. Peduto and R. Bryant, Neural responses to masked fear faces: sex differences and trauma exposure in posttraumatic stress disorder, J Abnormal Psychol 119 (2010), 241-247.

[16] A. Iwasaki-Sekin, A. Mano-Otagiri, H. Ohata, N. Yamauchi and T. Shibasaki, Gender differences in corticotrophin and corticosterone secretion and corticotrophin-releasing factor $\mathrm{mR}$ NA expression in the paraventricular nucleus of the hypothalamus and the central nucleus of the amygdale in response to footshock stress or psychological stress in rats, Psychoendocrinology 34 (2009), 226-237.

[17] V. Viau, B. Bingham, J. Davis, P. Lee and M. Wong, Gender and puberty interact on the stress-induced activation of parvocellular neurosecretory neurons and corticotrophin-releasing hormone mRMA expression in the rat, Endoctinology 146 (2005), 137-146.

[18] M. Weinstock, M. Razin, D. Schorer-Apelbaum, D. Men and R. McCarty, Gender differences in sympathoadrenal activity in rats at rest and in response to footshock stress, Int J Dev Neurosci 16 (1998), 289-295.

[19] B. Asia, R. Tordera, B. Lashers, J. Del Rio and R.M.J, Effects of maternal separation on hypothalamic-pituitary-adrenal responses, cognition and vulnerability to stress in adult female rats, Neuroscience 154 (2008), 1218-1226.

[20] V. Luine, Sex differences in chronic stress effects on memory in rats, Stress 5 (2002), 205-216.

[21] V. Critchlow, R.A. Liebelt, M. Bar-Sela, W. Mountcastle and H.S. Lipscomb, Sex difference in resting pituitary-adrenal function in the rat, Am J Physiol 205 (1963), 807-815.

[22] J.I. Kitay, Sex differences in adrenal cortical secretion in the rat, Endocrinol 68 (1961), 818-824.

[23] L.H. Burgess and R.J. Handa, Chronic estrogen-induced alterations in adrenocorticotropin and corticosterone secretion, and glucocorticoid receptor-mediated functions in female rats, Endocrinology 131 (1992), 1261-1269.

[24] R.J. Handa, L.H. Burgess, J.E. Kerr and J.A. O'Keefe, Gonadal steroid hormone receptors and sex differences in the hypothalamo-pituitary-adrenal axis, Horm Behav 28 (1994), 464-476.

[25] P. Palanza, Animal models of anxiety and depression: how are females different? Neurosci Biobehav Rev 25 (2001), 219233.

[26] M. Weinstock, Gender differences in the effects of prenatal stress on brain development and behaviour, Neurochem Res 32 (2007), 1730-1740.

[27] R. Gorski, J. Gordon, J. Shryne and A. Southam, Evidence for a morphological sex difference within the medial preoptic area of the rat brain, Brain Res Bull 148 (1978), 333-346.

[28] L.W. Christensen, D.M. Nance and G.R.A., Effects of hypothalamic and preoptic lesions on reproductive behavior in male rats, Brain Research Bulletin 2 (1977), 137-141.

[29] E. Davis, P. Popper and R. Gorski, The role of apoptosis in sexual differentiation of the rat sexually dimorphic nucleus of the preoptic area, Brain Res Bull 734 (1996), 10-18.

[30] M. Diamond, R. Johnson, D. Young and S. Singh, Age-related morphologic differences in the rat cerebral cortex and hippocampus: male-female; right-left, Exp Neurol 81 (1983), $1-13$.

[31] D. Fleming, R. Anderson, R. Rhees, E. Kinghorn and J. Bakaitis, Effects of prenatal stress on sexually dimorphic asymmetries in the cerebral cortex of the male rat, Brain Res Bull 16 (1986), 395-398.

[32] H.E. Jones, M.A. Ruscio, L.A. Keyser, C. Gonzalez, B. Billack, R. Rowe, C. Hancock, K.G. Lambert and C.H. Kinsley, 
Prenatal stress alters the size of the rostral anterior commissure in rats, Brain Res Bull 42 (1997), 341-346.

[33] C. Isgor and D.R. Sengelaub, Prenatal gonadal steroids affect adult spatial behavior, CA1 and CA3 pyramidal cell morphology in rats, Hormones and Behavior 34 (1998), 183-198.

[34] R.D. Romeo, E.M. Waters and B.S. McEwen, Steroid-induced hippocampal synaptic plasticity: sex differences and similarities, Neuron Glia Biol 1 (2004), 219-229.

[35] N.M. Conejo, H. Gonzalez-Pardo, C. Pedraza, F.F. Navarro, G. Vallejo and J.L. Arias, Evidence for sexual difference in astrocytes of adult rat hippocampus, Neurosci Lett 339 (2003), 119-122.

[36] J.A. Markham, K.P. McKian, T.S. Stroup and J.M. Juraska, Sexually dimorphic aging of dendritic morphology in CA1 of hippocampus, Hippocampus 15 (2005), 97-103.

[37] E. Gould, A. Westlind-Danielsson, M. Frankfurt and B.S. McEwen, Sex differences and thyroid hormone sensitivity of hippocampal pyramidal cells, Journal of Neuroscience $\mathbf{1 0}$ (1990), 996-1003.

[38] R.L. Roof, The dentate gyrus is sexually dimorphic in prepubescent rats: testosterone plays a significant role, Brain Research Bulletin 610 (1993), 148-151.

[39] G. Tabibnia, B.M. Cooke and S.M. Breedlove, Sex difference and laterality in the volume of mouse dentate gyrus granule cell layer, Brain Research 827 (1999), 41-45.

[40] A. Parducz and L.M. Garcia-Segura, Sexual differences in the synaptic connectivity in the rat dentate gyrus, Neuroscience Letters 161 (1993), 53-56.

[41] J.P. Andrade, M.D. Madeira and M.M. Paula-Barbosa, Sexual dimorphism in the subiculum of the rat hippocampal formation, Brain Research 875 (2000), 125-137.

[42] T.J. Shors, C. Chua and J. Falduto, Sex differences and opposite effects of stress on dendritic spine density in the male versus female hippocampus, Journal of Neuroscience 21 (2001), 6292-6297.

[43] C. Woolley and B. McEwen, Estradiol mediates fluctuation in hippocampal synapse density during the estrous cycle in the adult rat, J Neurosci 12 (1992), 2549-2554.

[44] E. Gould, C. Woolley, M. Frankfurt and B. McEwen, Gonadal steroids regulate dendritic spine density in hippocampal pyramidal cells in adulthood, J Neurosci 10 (1990), 1286-1291.

[45] C. Li, W. Brake, R. Romeo, J. Dunlop, M. Gordon, R. Buzescu, A. Magarinos, P. Allen, P. Greengard, V. Luine and B. McEwen, Estrogen alters hippocampal dendritic spine shape and enhances synaptic protein immunoreactivity and spatial memory in female mice, Proc Natl Acad Sci USA 101 (2004), 2185-2190.

[46] N. MacLusky, V. Luine, T. Hajszan and C. Leranth, The 17alpha and 17 beta isomers of estradiol both induce rapid spine synapse formation in the CA1 hippocampal subfield of ovariectomized female rats, Endocrinology 146 (2005), 287293.

[47] C. Smith and L. McMahon, Estrogen-induced increase in the magnitude of long-term potentiation occurs only when the ratio of NMDA transmission to AMPA transmission is increased, J Neurosci 25 (2005), 7780-7791.

[48] C. Woolley, H. Wenzel and P. Schwartzkroin, Estradiol increases the frequency of multiple synapse boutons in the hippocampal CA1 region of the adult female rat, J Comp Neurol 373 (1996), 108-117.

[49] M. Yankova, S. Hart and C. Woolley, From the cover: estrogen increases synaptic connectivity between single presynaptic inputs and multiple postsynaptic CA1 pyramidal cells: a serial electronmicroscopic study, Proc Natl Acad Sci USA 98 (2001), 3525-32530.

[50] E.A. Nimchinsky, B.L. Sabatini and K. Svoboda, Structure and function of dendritic spines, Annual Review of Physiology 64 (2002), 313-353.

[51] B. McEwen, Estrogen actions throughout the brain, Recent Prog Horm Res 57 (2002), 357-384.

[52] C. Leranth, O. Petnehazy and N.J. MacLusky, Gonadal hormones affect spine synaptic density in the CA1 hippocampal subfield of male rats, Journal of Neuroscience 23 (2003), 1588-1592.

[53] C. Lewis, B.S. McEwen and M. Frankfurt, Estrogen-induction of dendritic spines in ventromedial hypothalamus and hippocampus: effects of neonatal aromatase blockade and adult GDX, Developmental Brain Research 87 (1995), 91-95.

[54] J.M. Daniel and G.P. Dohanich, Acetylcholine mediates the estrogen-induced increase in NMDA receptor binding in CA1 of the hippocampus and the associated improvement in working memory, Journal of Neuroscience 21 (2001), 6949-6956.

[55] N. Weiland, Estradiol selectively regulates agonist binding sites on the N-methyl-D-aspartate receptor complex in the CA1 region of the hippocampus, Endocrinology 131 (1992), 662-668.

[56] C.S. Woolley and B.S. McEwen, Estradiol regulates hippocampal dendritic spine density via an N-methyl-D-aspartate receptordependent mechanism, Journal of Neuroscience 14 (1994), 7680-7687.

[57] B.S. McEwen and R.E. Brinton, Neuroendocrine aspects of adaptation, Prog. Brain Res 72 (1987), 11-26.

[58] A. Mazor, M.A. Matar, Z. Kaplan, N. Kozlovsky, J. Zohar and H. Cohen, Gender-related qualitative differences in baseline and post-stress anxiety responses are not reflected in the incidence of criterion-based PTSD-like behaviour patterns, World J Biol Psychiatry 10 (2009), 856-869.

[59] H. Louvart, S. Maccari, J. Lesage, M. Leonhardt, A. DickesCoopman and M. Darnaudéry, Effects of a single footshock followed by situational reminders on HPA axis and behaviour in the aversive context in male and female rats, Psychoneuroendocrinology 31 (2006), 92-99.

[60] D.D. Rasmussen, N.J. Crites and B.L. Burke, Acoustic startle amplitude predicts vulnerability to develop post-traumatic stress hyper-responsivity and associated plasma corticosterone changes in rats, Psychoneuroendocrinology 33 (2008), 282291.

[61] V. Viau and M.J. Meaney, Testosterone-dependent variations in plasma and intrapituitary corticosteroid binding globulin and stress hypothalamic-pituitary-adrenal activity in the male rat, J Endocrinol 181 (2004), 223-231.

[62] J. Le Mevel, S. Abitbol, G. Beraud and J. Maniey, Temporal changes in plasma adrenocorticotropin concentration after repeated neurotropic stress in male and female rats, Endocrinology 105 (1979), 812-817.

[63] A.L. Herbst, F.E. Yates, D.W. Glenister and J. Urquhart, Variations in hepatic inactivation of corticosterone with changes in food intake: an explanation of impaired corticosteroid metabolism following noxious stimuli, Endocrinology 67 (1960), 222-238.

[64] A.A. Hagen and R.C. Troop, Influence of age, sex and adrenocortical status on hepatic reduction of cortisone in vitro, Endocrinology 67 (1960), 194-203.

[65] R.C. Troop, Influence of gonadal hormones on the metabolism of cortisone, Endocrinology 64 (1959), 671-675.

[66] J. Urquhart, F.E. Yates and A.L. Herbst, Hepatic regulation of adrenal cortical function, Endocrinology 64 (1959), 816-830. 
[67] C. Rivier, Gender, sex steroids, corticotrophin-releasing factor, and HPA response to stress, Biochem Behv (1999), 739-751.

68] C.A. Gemzell, [Increase in the formation and the secretion of ACTH in rats following administration of oestradiolmonobenzoate.], Acta Endocrinol (Copenh) 11 (1952), 221228.

[69] D. Gompertz, The effect of sex hormones on the adrenal gland of the male rat, J Endocrinol 17 (1958), 107-113.

[70] L.H. Burgess and R.J. Handa, Hormonal regulation of androgen receptor mRNA in the brain and anterior pituitary gland of the male rat, Brain Res Mol Brain Res 19 (1993), 31-38.

[71] M.P. Carey, C.H. Deterd, J. de Koning, F. Helmerhorst and E.R. de Kloet, The influence of ovarian steroids on hypothalamic-pituitary-adrenal regulation in the female rat, $J$. Endocrinol 144 (1995), 311-321.

[72] A. Peiffer and N. Barden, Estrogen-induced decrease of glucocorticoid receptor messenger ribonucleic acid concentration in rat anterior pituitary gland, Mol Endocrinol 1 (1987), 435440.

[73] B.B. Turner, Sex difference in glucocorticoid binding in rat pituitary is estrogen dependent, Life Sci 46 (1990), 1399-1406.

[74] V.K. Patchev, S. Hayashi, C. Orikasa and O.F. Almeida, Implications of estrogen-dependent brain organization for gender differences in hypothalamo-pituitary-adrenal regulation, FASEB J 9 (1995), 419-423.

[75] M.J. Weiser and R.J. Handa, Estrogen impairs glucocorticoid dependent negative feedback on the hypothalamic-pituitaryadrenal axis via estrogen receptor alpha within the hypothalamus, Neuroscience 159 (2009), 883-895.

[76] V.K. Patchev and O.F. Almeida, Gender specificity in the neural regulation of the response to stress: new leads from classical paradigms, Mol Neurobiol 16 (1998), 63-77.

[77] C.M. McCormick, W. Linkroum, B.J. Sallinen and N.W. Miller, Peripheral and central sex steroids have differential effects on the HPA axis of male and female rats, Stress 5 (2002), 235-247.

[78] M. Baum, Activational and organizational effects of estradiol on male behavioral neuroendocrine function, Scand J Psychol 44 (2003), 213-220.

[79] B. McEwen, Steroid hormones: effect on brain development and function, Horm Res 37 (1992), 1-10.

[80] J.H. Gaskin and J.I. Kitay, Hypothalamic and pituitary regulation of adrenocortical function in the hamster: effects of gonadectomy and gonadal hormone replacement, Endocrinology 89 (1971), 1047-1053.

[81] R.J. Handa, K.M. Nunley, S.A. Lorens, J.P. Louie, R.F. McGivern and M.R. Bollnow, Androgen regulation of adrenocorticotropin and corticosterone secretion in the male rat following novelty and foot shock stressors, Physiol Behav 55 (1994), 117-124.

[82] E.W. Bingaman, D.J. Magnuson, T.S. Gray and R.J. Handa, Androgen inhibits the increases in hypothalamic corticotropinreleasing hormone $(\mathrm{CRH})$ and $\mathrm{CRH}$-immunoreactivity following gonadectomy, Neuroendocrinology 59 (1994), 228-234.

[83] Y. Lin, C. Wesrenbroek, P. Bakker, J. Termeer, A. Liu, X. Li and G. Ter Horst, Effects of long-term stress and recovery on the prefrontal cortex and dentate gyrus in male and female rats, Cerebral Cortex 18 (2008), 2762-2774.

[84] R.M. Shansky, G. Bender and A. F. Arnsten, Estrogen prevents norepinephrine alpha-2a receptor reversal of stress-induced working memory impairment, Stress 12 (2009), 457-463.

[85] T. Poltyrev and M. Weinstock, Gender difference in the prevention of hyperanxiety in adult prenatally stressed rats by chronic treatment with amitriptyline, Psychopharmacology (Berl) 171 (2004), 270-276.

[86] Y. Masubuchi, T. Iwasa, S. Fujita, T. Suzuki, T. Horie and S. Narimatsu, Regioselectivity and substrate concentrationdependency of involvement of the CYP2D subfamily in oxidative metabolism of amitriptyline and nortriptyline in rat liver microsomes, J Pharm Pharmacol 48 (1996), 925-929.

[87] H. Cohen, Z. Joseph and M. Matar, The relevance of differential response to trauma in an animal model of post-traumatic stress disorder, Biol Psychiatry 53 (2003), 463-473.

[88] H. Cohen and J. Zohar, Animal models of post traumatic stress disorder: The use of cut off behavioral criteria, The Annals New-York Academy of Sciences 1032 (2004), 167-178.

[89] H. Cohen, J. Zohar, M.A. Matar, Z. Kaplan and A.B. Geva, Unsupervised fuzzy clustering analysis supports behavioral cutoff criteria in an animal model of posttraumatic stress disorder, Biol Psychiatry 58 (2005), 640-650.

[90] H. Cohen, J. Zohar, M.A. Matar, K. Zeev, U. Loewenthal and G. Richter-Levin, Setting apart the affected: the use of behavioral criteria in animal models of post traumatic stress disorder, Neuropsychopharmacology 29 (2004), 1962-1970.

[91] R. Andrew, D.I. Phillips and B.R. Walker, Obesity and gender influence cortisol secretion and metabolism in man, $J$ Clin Endocrinol Metab 83 (1998), 1806-1809.

[92] M. Balbo, R. Leproult and E. Van Cauter, Impact of sleep and its disturbances on hypothalamo-pituitary-adrenal axis activity, Int J Endocrinol 2010 (2010), 759234.

[93] T. Smeets, I. Dziobek and O.T. Wolf, Social cognition under stress: differential effects of stress-induced cortisol elevations in healthy young men and women, Horm Behav 55 (2009), 507-513.

[94] N. Takai, M. Yamaguchi, T. Aragaki, K. Eto, K. Uchihashi and Y. Nishikawa, Gender-specific differences in salivary biomarker responses to acute psychological stress, Ann $N Y$ Acad Sci 1098 (2007), 510-515.

[95] H. Vierhapper, P. Nowotny and W. Waldhausl, Sex-specific differences in cortisol production rates in humans, Metabolism 47 (1998), 974-976.

[96] R. Yehuda, A. McFarlane and A. Shalev, Predicting the development of posttraumatic stress disorder from the acute response to a traumatic event, Biological Psychiatry 44 (1998), 1305-1313.

[97] R. Yehuda, J. Flory, L. Pratchett, J. Buxbaum, M. Ising and F. Holsboer, Putative Biological Mechanisms for the Association between Early Life Adversity and the Subsequent Development of PTSD, Psychopharmacology (Berl) 212 (2010), 405-417. 


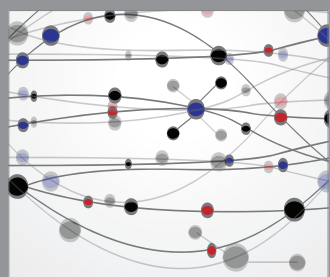

The Scientific World Journal
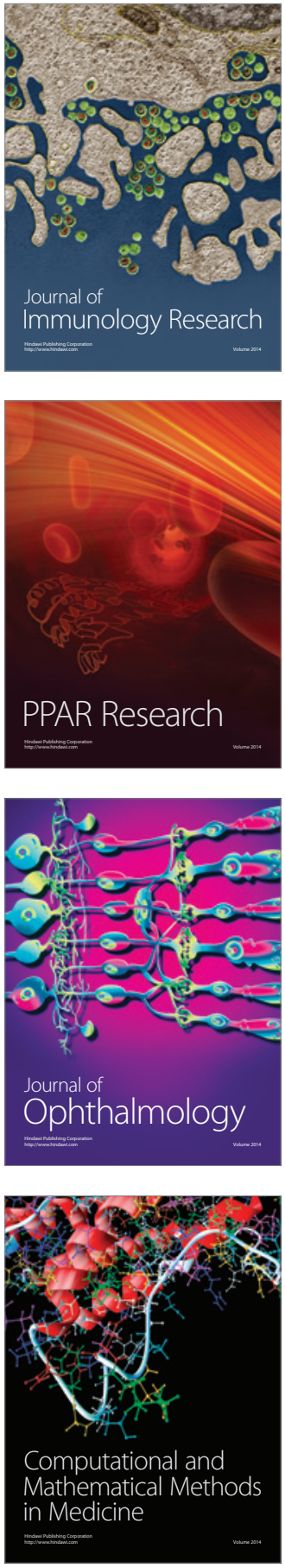

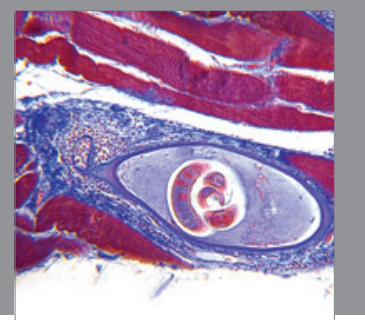

Gastroenterology

Research and Practice
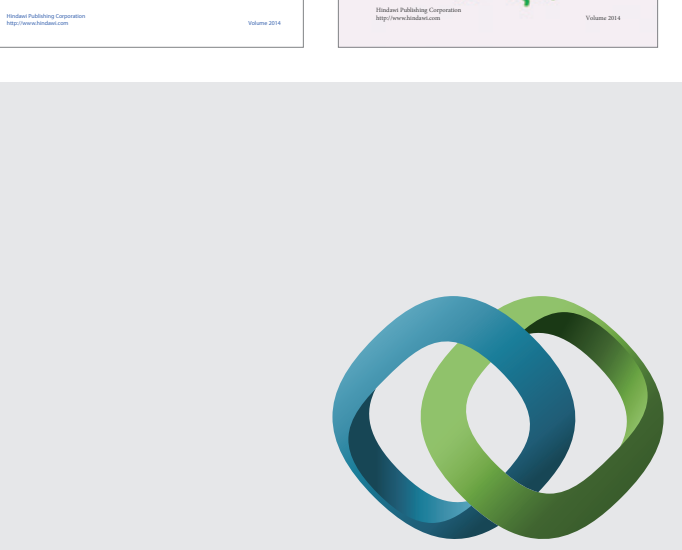

\section{Hindawi}

Submit your manuscripts at

http://www.hindawi.com
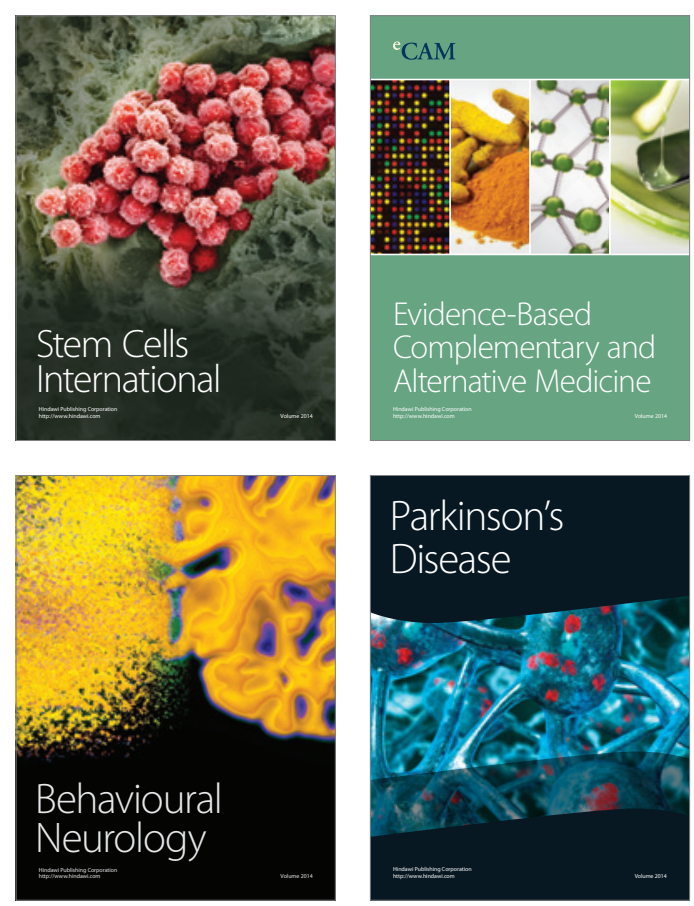

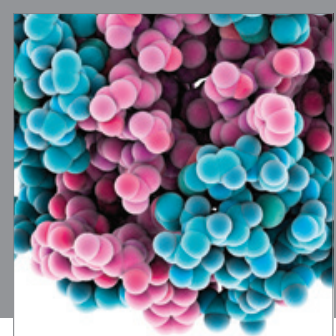

Journal of
Diabetes Research

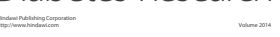

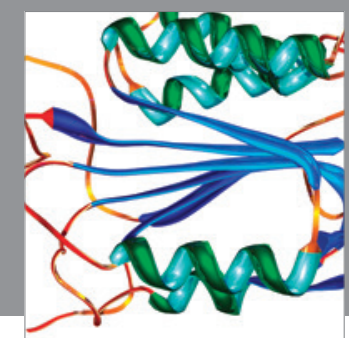

Disease Markers
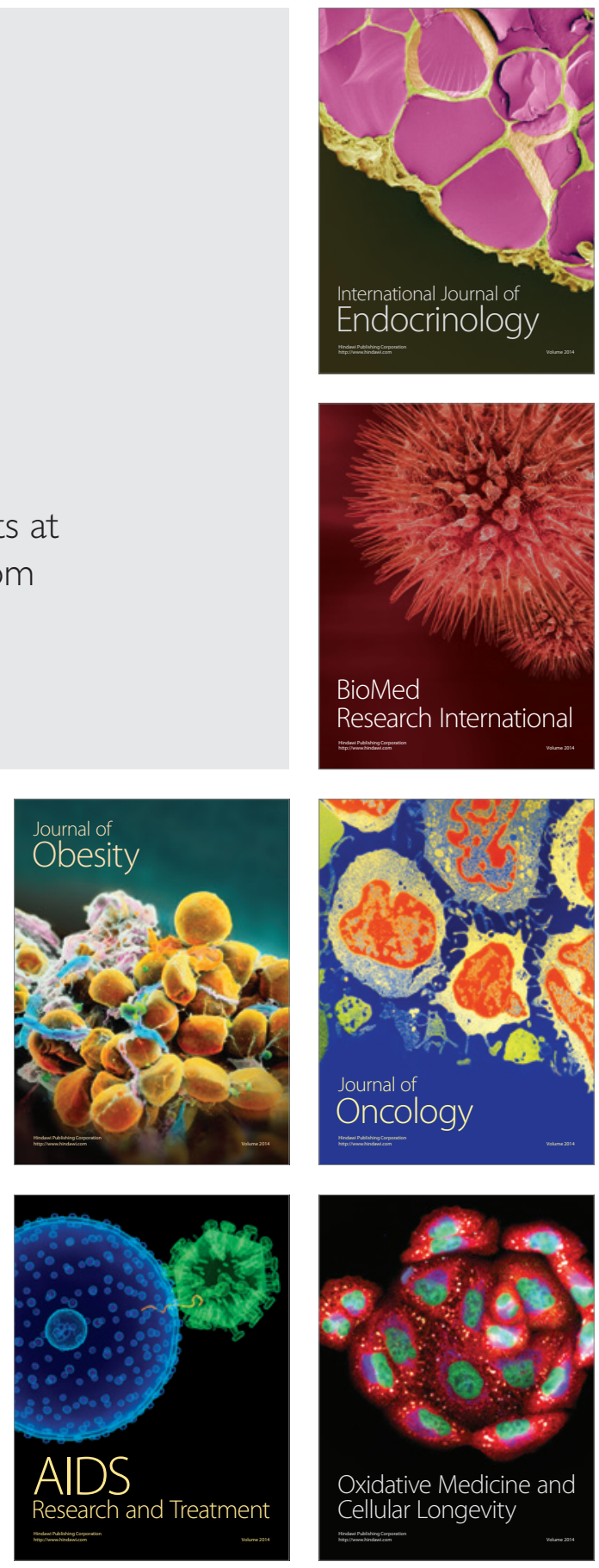